\title{
Telephone OSCE
}

\author{
C. V. Haldipur
}

Published online: 16 January 2014

(C) Academic Psychiatry 2014

To the editor:

Objective structured clinical examinations (OSCEs) are being used by licensing bodies such as the National Board of Medical Examiners, and most medical schools to evaluate students' clinical skills. This letter describes the modification of OSCEs to evaluate students' communication and clinical skills by using standardized patients who communicate their children's symptoms over the telephone. The student is expected to give advice after asking appropriate questions about medical history and obtaining other relevant information. There are no reports in the literature about this method of examination.

The impetus for this was a contretemps of a standardized patient who failed to show up due to inclement weather. Since the examination could not be postponed, it was agreed that the standardized patient could stay home and accept telephone calls from students. The students were told to listen to the caller's concern, ask appropriate questions, and then give advice.

It soon dawned on us that telephone OSCEs could be used in addition to the standard method of testing students. Furthermore, children and adolescents cannot, for obvious reasons, be available as standardized patients. We asked child psychiatrists to give us some typical telephone calls that they had received from patients or parents. A retired health care

worker agreed to be the standardized patient accepting telephone calls from students.

Four complaints were selected on the basis of their ability to test the student's competence to deal with issues relating to pharmacology, emergency situations such as a suicidal attempt, parental concern about press reports about side or adverse effects of medication, and finally, substance use by an adolescent.

As we gained more experience, we were able to add and modify the telephone calls. The standardized patient, too, was able to get more sophisticated in evaluating students' performance: Was the student polite? Was the advice given in a professional manner? Did the student appear to be listening and empathic? And finally, was the advice appropriate for the circumstance?

This test was used in addition to the conventional observed standardized clinical encounter. Its usefulness is obvious when one considers that doctors spend a lot of time dealing with clinical situations over the phone. An added advantage is that it obviates the need for standardized children and adolescent patient. Unfortunately, the test was discontinued due to fiscal and organizational concerns. It remains useful as an additional assessment tool.

C. V. Haldipur $(\bowtie)$

Upstate Medical University, Syracuse, NY, USA

e-mail: haldipuc@upstate.edu 